\title{
Damage Identification in Footbridges from Natural
}

\section{Frequency Measurements}

\author{
Ivana Mekjavić ${ }^{1}$ and Domagoj Damjanović ${ }^{2}$ \\ 1. Department of Structures, Faculty of Civil Engineering, University of Zagreb, Zagreb 10000, Croatia \\ 2. Department of Engineering Mechanics, Faculty of Civil Engineering, University of Zagreb, Zagreb 10000, Croatia
}

\begin{abstract}
The present study aims to develop a robust structural damage identification method that can be used for the evaluation of bridge structures. An approach for the structural damage identification based on the measurement of natural frequencies is presented. The structural damage model is assumed to be associated with a reduction of a contribution to the element stiffness matrix equivalent to a scalar reduction of the material modulus. A computational procedure for the direct iteration technique based on the non-linear perturbation theory is proposed to identify structural damage. The presented damage identification technique is applied to the footbridge over the Slunjcica River near Slunj to demonstrate the effectiveness of the proposed approach. Using a limited number of measured natural frequencies, reduction in the stiffness of up to $100 \%$ at multiple sites is detected. The results indicate that the proposed approach can be successful in not only predicting the location of damage but also in determining the extent of structural damage.
\end{abstract}

Key words: Footbridges, damage identification, natural frequency measurements.

\section{Introduction}

Damage or fault detection, as determined by changes in the dynamic properties or response of structures, is a subject that has received considerable attention in the literature. Since the changes in the stiffness of the structure, whether local or distributed, will cause changes in the modal parameters (notably natural frequencies, mode shapes, etc.), the location and the severity of damage in structure can be determined by changes in the modal characteristics. Furthermore, since the natural frequencies are rather easy to measure at a relatively high level of accuracy, the methods based on the measurements of natural frequencies are potentially attractive [1-5]. These characteristics can be obtained by measurements at one point of the structure and are independent of the location chosen. Recently, various methods for damage identification based on vibration

Corresponding author: Ivana Mekjavić, associate professor, research fields: bridges, concrete structures and dynamics. E-mail: ivanam@grad.hr. measurements have been proposed, such as perturbation methods [4], sensitivity analysis methods [6], model updating techniques [7] and neural network methods [8].

Bicanic and Chen [4] proposed a novel perturbation-based approach using the exact relationship between the changes of structural parameters and the changes of modal parameters in order to avoid the insufficiency of the first-order sensitivity analysis. For damage detection, the first-order approximation may be inaccurate since a large change of structural parameters due to damage might need to be detected [1-3].

In this paper, the direct iteration technique based on the non-linear perturbation theory is utilized to identify structural damage, while only natural frequencies for the damaged structure are required. The effectiveness of the proposed numerical procedure was already demonstrated through an example of the real concrete girder bridge with simulated damage, laboratory testing of a simply supported reinforced concrete beam subjected to 
various levels of static load [9], and finally full scale testing of six concrete girder bridges of different ages in Croatia [10].

This research attempts to provide an effective and practical damage identification approach for real large scale engineering structures, such as footbridges. Here, the application of the proposed technique to structural inspection by vibration frequencies monitoring is described in the following. It is shown that the proposed approach performs well for predictions of both the location and the extent of structural damage even when only a limited number of measured natural frequencies are available, which is particularly useful for large scale structures.

\section{Direct Iteration Technique}

The computational procedure for the direct iteration technique has been developed to solve for the element scalar damage parameters $\alpha_{j}$ as well as the mode participation factors $C_{i k}$ [4]. The iterative solution procedure is described in the following section.

Depending on the number of available natural frequencies for the damaged structure $N F$ (number of equations) and the number of structural damage parameters NXE (number of unknows), the eigenmode-stiffness sensitivity matrix $S_{i j}$ may not be square. Theoretically, if the number of available natural frequencies $N F$ is equal to $N X E$, a solution may be determined exactly. However, only a smaller number of natural frequencies can usually be measured. Hence, the number of the measured natural frequencies for the damaged structure $N F$ is less than the number of structural damage parameters (finite-elements, $N F<N X E$ ), which renders the equations underdetermined, i.e., ill-conditioned.

In order to find a solution for what is in general an ill-conditioned system, the SVD (singular value decomposition) technique is applied.

A FORTRAN computer program for structural damage identification has been developed based on the knowledge of the computational procedure presented below.

Computational procedure for the proposed technique is as follows:

(1) Step 1. Assuming the initial mode participation factors $C_{i k}^{0}$ to be 0 , i.e., there are no changes in eigenvectors. Establish the initial values for $\alpha_{j}^{1}$ and $C_{i k}^{1}$ from

$$
\sum_{j=1}^{N X E} S_{i j}^{1} \alpha_{j}^{1}=z_{i}
$$

where, $S_{i j}$ and $z_{i}$ are the individual elements of the eigenmode-stiffness sensitivity matrix and vector, respectively, which are defined as

$$
\begin{gathered}
S_{i j}^{1}=a_{i j i}=\boldsymbol{\phi}_{i}^{\mathrm{T}} \boldsymbol{K}_{j} \boldsymbol{\phi}_{i} \\
z_{i}=\Delta \lambda_{i}
\end{gathered}
$$

where, $a_{i j i}$ is the eigenmode-stiffness sensitivity coefficient; $\boldsymbol{\phi}_{i}$ is the $i$ th original eigenvector; $\boldsymbol{K}_{j}$ is the contribution of the $j$ th element to the global stiffness matrix; $\Delta \lambda_{i}$ is the change in the $i$ th eigenvalue;

$$
C_{i k}^{1}=\frac{b_{k i}^{1}}{\lambda_{i}^{*}-\lambda_{k}-b_{k k}^{1}}
$$

where, $b_{k k}$ and $b_{k i}$ can be defined in general form as

$$
b_{k i}^{1}=\sum_{j=1}^{N X E} a_{k j i} \alpha_{j}^{1}
$$

$\lambda_{k}$ is the $k$ th original eigenvalue and a superscript refers to the damaged structure;

(2) Step 2. Evaluate current estimate for $\alpha_{j}^{n}$ from

$$
\sum_{j=1}^{N X E} S_{i j}^{n} \alpha_{j}^{n}=z_{i}
$$

where,

$$
S_{i j}^{n}=a_{i j i}+\sum_{l=1, l \neq i}^{N M} C_{i l}^{n-1} a_{i j l}
$$

and $N M$ is the number of the original eigenvectors available;

(3) Step 3. Evaluate new modal participation factors $C_{i k}^{n}$ from

$$
C_{i k}^{n}=\frac{b_{k i}^{n}+\sum_{l=1, l \neq i, k}^{N M} C_{i l}^{n-1} b_{k l}^{n}}{\lambda_{i}^{*}-\lambda_{k}-b_{k k}^{n}}
$$

where, $b_{k k}, b_{k i}$ and $b_{k l}$ can be defined in general form as 


$$
b_{k i}^{n}=\sum_{j=1}^{N X E} a_{k j i} \alpha_{j}^{n}
$$

and return to Step 2 if solution has not converged.

\section{Experimental and Numerical Study on a Footbridge}

\subsection{Description of Bridge and Dynamic Testing}

The tested bridge over the Slunjcica River near Slunj in Croatia is a six-span simply supported steel girder bridge. The spans of the bridge are $8.5+8.5+$ $11.8+11.5+12.0+11.5=63.8 \mathrm{~m}$. The superstructure consists of four steel girders, $90 \mathrm{~cm}$ high, connected by truss cross girders at the supports and in the fourth of spans and an orthotropic deck made of omega-shaped cross profiles. The abutments and five piers are constructed from masonry stones. The longitudinal disposition and the cross section of the footbridge are shown in Fig. 1.

After completion of the concrete arch bridge over the Slunjcica River in the 1960s, this steel bridge remained open only for pedestrian traffic.
In experimental study of the bridge, OMA (operational modal analysis) was implemented using ambient environmental and traffic excitation. This simplifies the testing procedure as only response measurement is required for determination of natural frequencies, modal shapes and damping ratios.

Identification of modal parameters was conducted using methods of FDD (frequency domain decomposition). The procedure is based on SVD of PSD (power spectral density) matrix of the measured responses $\left(\boldsymbol{G}_{\boldsymbol{y} y}\right)$. It is assumed that the loading is white noise process, the structure is lightly damped and close mode shapes are geometrically orthogonal [11]. Result of SVD is:

$$
\hat{G}_{y y}\left(\omega_{i}\right)=U_{i} S_{i} U_{i}^{H}
$$

where, $\boldsymbol{U}_{\boldsymbol{i}}$ represents unitary matrix of singular vectors, and $\boldsymbol{S}_{\boldsymbol{i}}$ diagonal matrix of singular values. Eq. (10) is known at discrete frequencies $\omega=\omega_{i}$. At the discrete frequency of the resonance peak, the first singular vector is an estimate of mode shape [12].

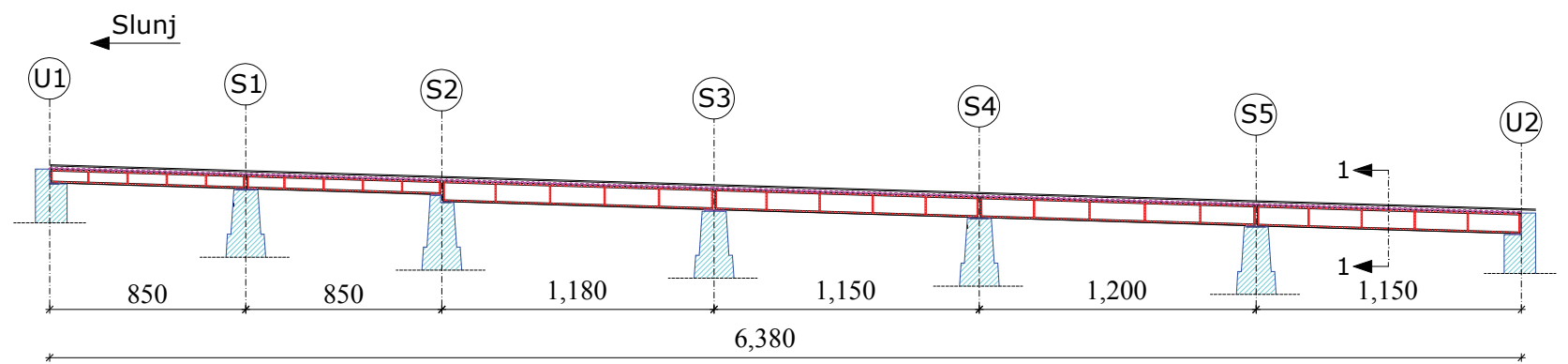

(a)

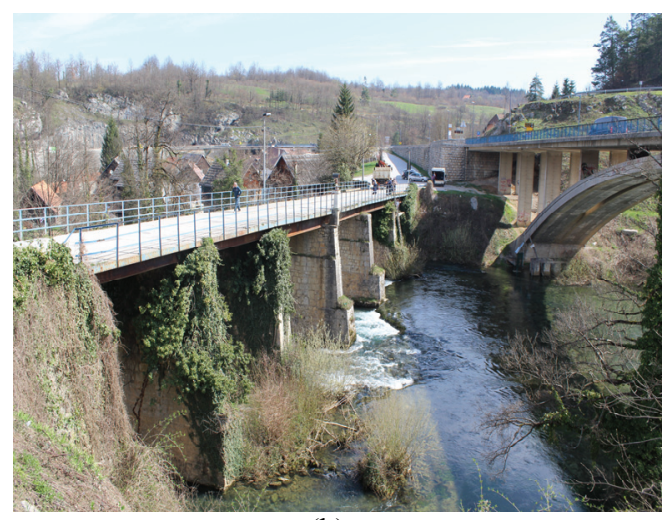

(b)

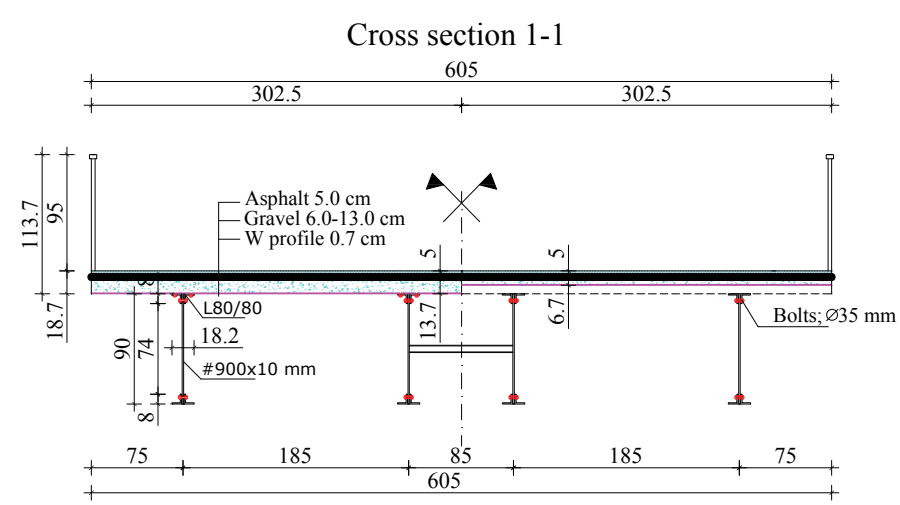

(c)

Fig. 1 A footbridge over Slunjcica River near Slunj, Croatia: (a) elevation; (b) view; (c) cross-section (units in cm). 
EFDD (enhanced frequency domain decomposition) identifies the SDOF (single degree of freedom) PSD function around the peak by comparing vector at the peak with the vectors corresponding to discrete frequencies around the peak using MAC (modal assurance criterion). Inverse discrete Fourier transform of acquired SDOF function enables determination of damping ratio for that particular mode [13].

CFDD (curve-fitting frequency domain decomposition) technique is similar to EFDD but it applies curve-fitting to the SDOF function directly in the frequency domain [14].

The dynamic response of each simple span was measured with six accelerometers connected to a PC based data acquisition system (Brüel\&Kjær 3560C). The layout of the accelerometers is shown in Fig. 2. All data were recorded in the notebook computer and analysis was performed using pulse operational modal analysis software.

Fig. 3 shows singular values of spectral density matrices and SDOF function of the first four vertical bending modes. Mode shapes were estimated as first singular vectors at the resonance peak. Natural frequencies and damping ratios for the first four vertical bending modes are shown in Table 1, as well as standard deviations of measured natural frequencies determined on the basis of six responses. Standard deviations of measured frequencies are all within 1\% which suggests very good accuracy of measurement.

The experimental natural frequencies were then used in the direct iteration technique (Step 1 in Section 2) to calculate the extent of damage for each of finite-elements (structural damage parameters).

\subsection{Finite Element Model}

The 3D FE (three-dimensional finite-element) model of the footbridge is developed using the Sofistik software. Since the bridge superstructure is simply supported, one span of the bridge is modelled. Two types of elements were used to model the 3D bridge superstructure, beam and quadrilateral (quad) elements. Quadrilateral mesh was used to simulate both the gravel and asphalt layers on the bridge deck. The boundary conditions at the end nodes of simply supported steel beams are specified by suppressing the corresponding degrees of freedom. The need for

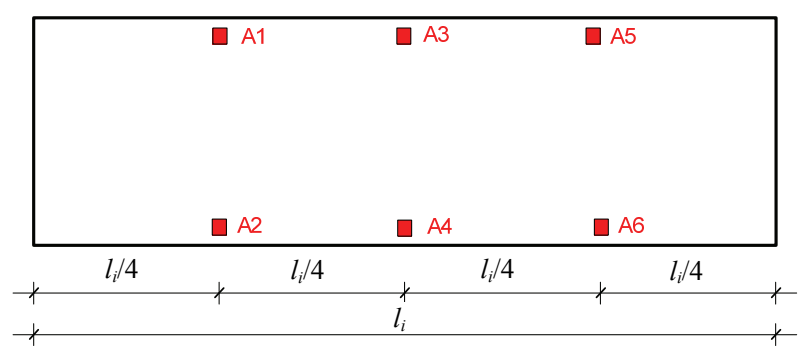

Fig. 2 Locations of accelerometers for each simple span of length $l_{i}$.

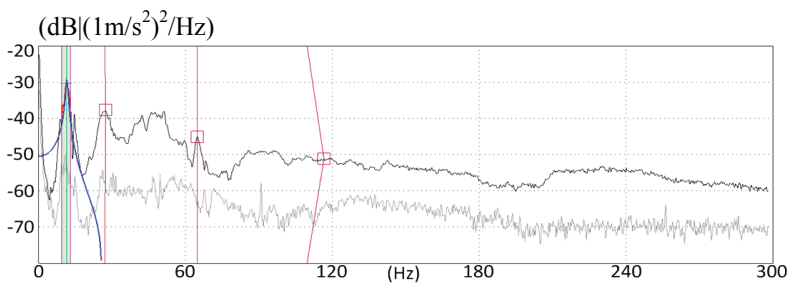

(a)

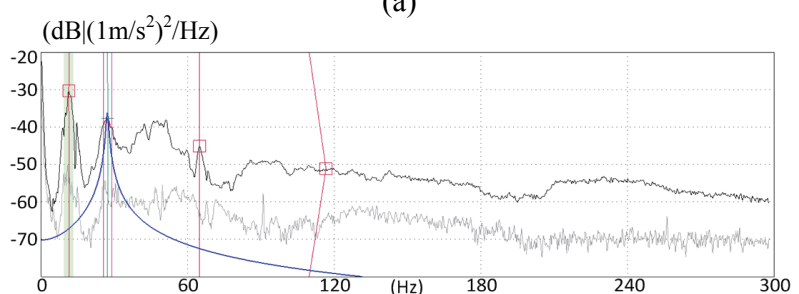

(b)

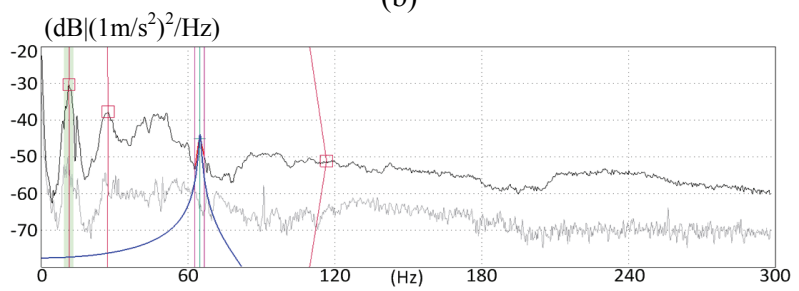

(c)

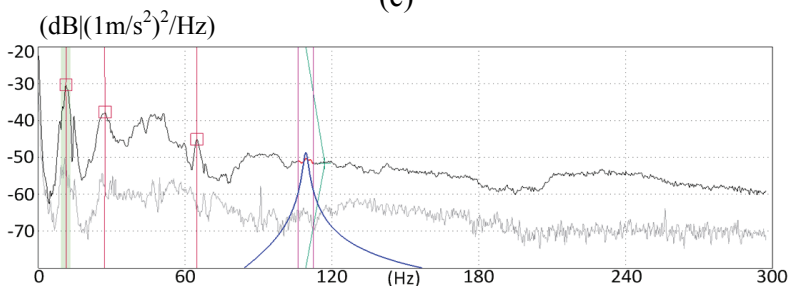

(d)

Fig. 3 Singular values of PSD matrices: (a) 1st bending mode $(8.62 \mathrm{~Hz})$; (b) 2nd bending mode $(28.74 \mathrm{~Hz})$; (c) 3rd bending mode $(69.17 \mathrm{~Hz}) ;$ (d) 4 th bending mode (114.40 Hz). 
Table 1 Experimentally determined natural frequencies and damping ratios.

\begin{tabular}{lcl}
\hline Mode & $\begin{array}{l}\text { Natural frequency } \pm \text { standard } \\
\text { deviation }(\mathrm{Hz})\end{array}$ & Damping ratio (\%) \\
\hline 1 & 8.62 & 3.0550 \\
2 & 28.74 & 1.7600 \\
3 & 69.17 & 0.7373 \\
4 & 114.40 & 1.2189 \\
\hline
\end{tabular}

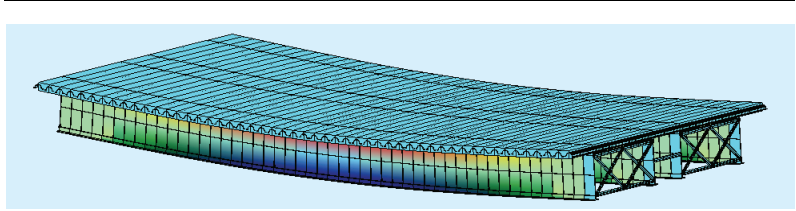

(a)

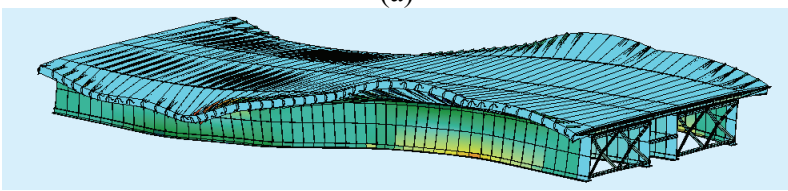

(b)

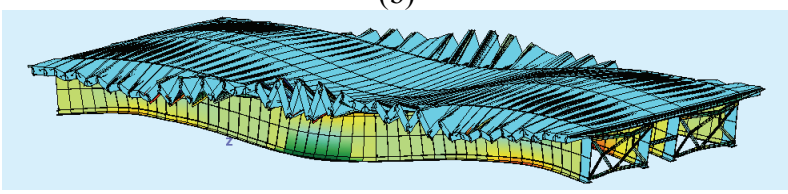

(c)

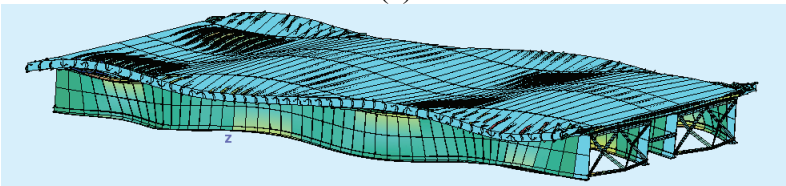

(d)

Fig. 4 First four vertical bending modes of 3D FE model (Sofistik): (a) 1st bending mode $(8.798 \mathrm{~Hz})$; (b) 2nd bending mode $(29.102 \mathrm{~Hz})$; (c) 3rd bending mode $(74.418 \mathrm{~Hz})$; (d) 4th bending mode (122.302 Hz).

Table 2 Finite-element natural frequencies for undamaged bridge.

\begin{tabular}{lccc}
\hline Mode & $\begin{array}{l}\text { 3D FE } \\
\text { model }(\mathrm{Hz})\end{array}$ & $\begin{array}{l}\text { 2D FE } \\
\text { model }(\mathrm{Hz})\end{array}$ & $\begin{array}{l}\text { Relative } \\
\text { error }(\%)\end{array}$ \\
\hline 1 & 8.798 & 8.799 & -0.01 \\
2 & 29.102 & 35.266 & -17.48 \\
3 & 74.418 & 79.933 & -6.90 \\
4 & 122.302 & 147.884 & -17.30 \\
\hline
\end{tabular}

kinematic constraints (dependent degrees of freedom) arises for rigidly connected nodes. The elasticity modulus of steel assumed for the un-tuned FE model was as the design one $E=2.1 \times 10^{8} \mathrm{kN} / \mathrm{m}^{2}$. The first four bending mode shapes of the model are shown in Fig. 4.

The natural frequencies for the undamaged bridge determined from the finite-element analyses are summarized in Table 2. The comparison with the test results is not possible since the measured natural frequencies for the undamaged bridge are not available. As expected, the natural frequencies for the damaged structure decreases as the structural stiffness decreases and the mass of the structure stays the same.

\subsection{Damage Identification}

Based on the natural frequencies for the undamaged and the damaged structure, a direct iteration technique was employed for structural damage identification.

The computer program developed here for dynamic analysis and damage identification is for the solution of 2D (two-dimensional) framed structures and uses beam-column elements. These elements each have 6 degrees of freedom, incorporating two translations and a rotation at each node, although the axial displacement is ignored in this example.

In order to avoid problems associated with structural symmetry, six non-symmetric finite-elements with seven nodes and a total of 12 degrees of freedom are generated (Fig. 5).

All structural members have the same material properties with experimentally estimated elastic modulus $E=2.1 \times 10^{8} \mathrm{kN} / \mathrm{m}^{2}$ and density $\rho=7.85 \mathrm{t} / \mathrm{m}^{3}$ and the same cross sectional area $A=0.3277 \mathrm{~m}^{2}$ and second moment of area $I=7.9687 \times 10^{-3} \mathrm{~m}^{4}$.

The first four vertical natural frequencies for the undamaged structure are listed in Table 2.

The information about four measured natural frequencies of the damaged structure (modified frequencies) is used and all original eigenvectors are considered to obtain the structural damage parameters for damaged footbridge, as shown in Fig. 6. The correlation between eigenvectors for the originally undamaged structure and the damaged structure is checked using the MAC factors. It seems that the modes for the damaged structure obtained from the direct iteration technique match the corresponding modes for the original structure very well.

The results of the damage prediction obtained from 


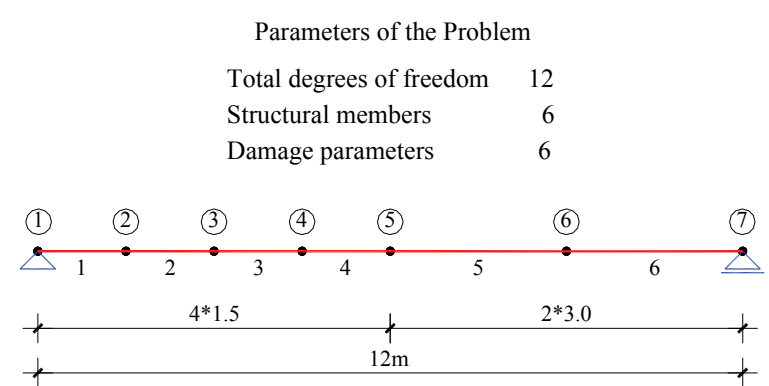

Fig. 5 One-span (of six-span) girder bridge, divided in six non-symetric finite-elements with seven nodes.

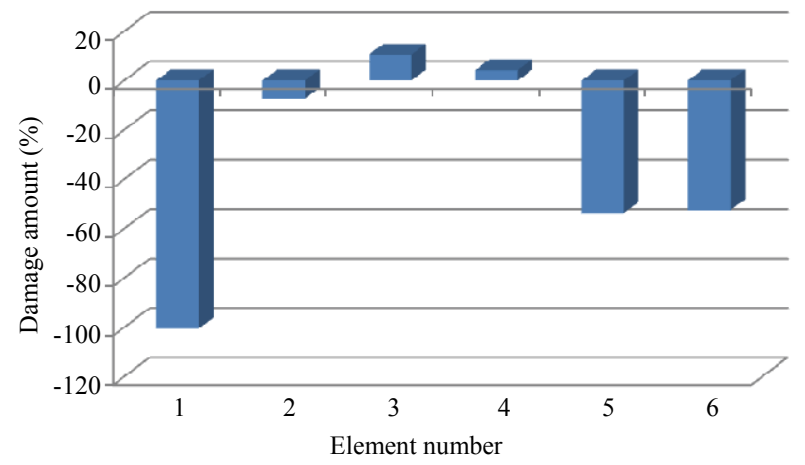

Fig. 6 Inverse damage predictions from direct iteration technique for footbridge near Slunj, four experimental frequencies used.

the direct iteration technique are presented in Fig. 6. At the horizontal axes in this figure, there are six parts of girder, i.e., six finite-elements. Those 6 parts of girder are positioned in the bridge structure according to Fig. 5. Damage amounts (\%) at vertical axes for every one of six parts of girder in Fig. 6 represents the change in structural stiffness expressed as the damage parameter $\alpha_{j}$ calculated by the computational procedure for the direct iteration technique presented in Step 1 in Section 2. A negative value in Fig. 6 represents the reduction in stiffness and positive value represents false change in the stiffness, which can never be produced by damage. From the results, it is concluded that the prediction of structural damage is significantly sensitive to the quality of the measured natural frequencies, which is caused by the ill-conditioned system of governing equations.

It is found that only a limited number of natural frequencies for the damaged structure are required, even four measured natural frequencies are sufficient to correctly predict structural damage.

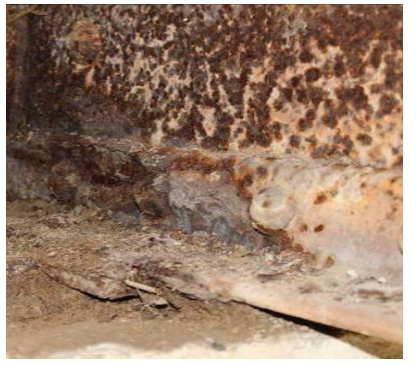

(a)

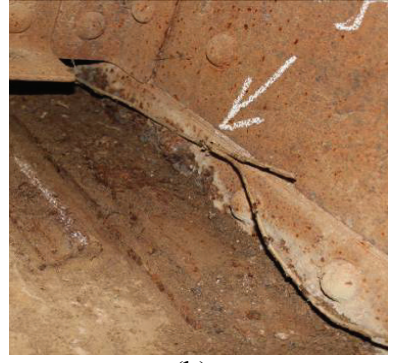

(b)

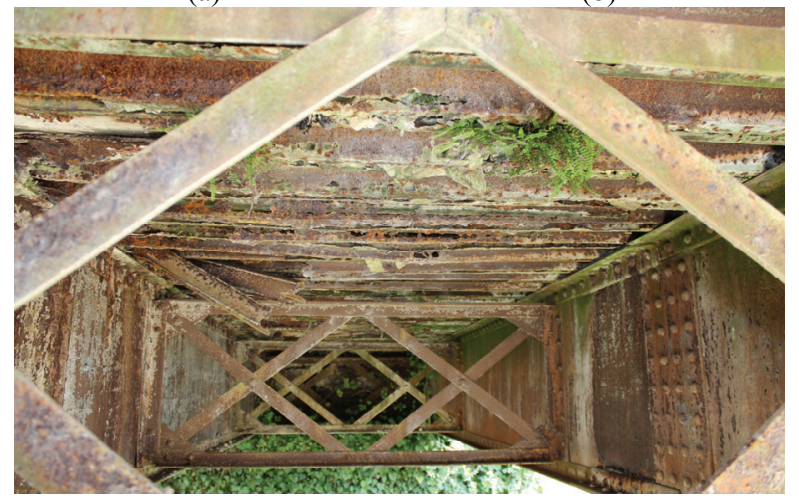

(c)

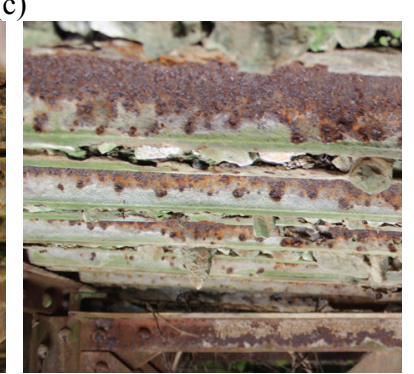

(e)
Fig. 7 Visual inspection: (a) heavy corrosion of bottom flange; (b) web of an I-girder end on a bearing; (c) truss cross girders; (d) and (e) severely corroded steel omega-shaped cross beams.

With an assumption that the stiffness degrades proportionally to the damage, i.e., the change in the stiffness matrix is expressed as the summation of changes proportional to the element matrices, the results show that structural stiffness decreases through the years. It is obvious that this footbridge has sustained a very serious damage (damage parameter $\alpha$ ranging up to $100 \%$ stiffness reduction).

Results of the damage identification procedure agreed well with the results of the visual inspection (Fig. 7) [15]. Several forms of damage, such as cracking, corrosion losses, delamination and changes in boundary conditions, cause a reduction in the stiffness of the damaged footbridge. 


\section{Conclusions}

The presented structural damage identification technique has shown its capability of successfully determining both the location and the size of damage from a limited number of measured natural frequencies for the damaged structure. The results show that the method performs well even for the structure with a very serious damage. The proposed method is also suitable for symmetric structures, if a non-symmetric element mesh is generated.

The practicability of the proposed method has been demonstrated by employing experimental measured natural frequencies and applying this method to real engineering structures, such as bridges. Although the use of the proposed technique here is illustrated for the simply supported footbridge, the same concept can be extended for various types of structures.

Furthermore, it is found that the presented approach is quite sensitive to the quality of the measured natural frequencies available for structural damage identification due to the nature of ill-conditioned system.

Finally, the results of this research indicate that the concept of a monitoring technique using the measured natural frequencies for the assessment of the current state of structures during their service life is feasible and practicable.

\section{References}

[1] Cawley, P., and Adams, R. D. 1979. "The Location of Defects in Structures from Measurements of Natural Frequencies." Journal of Strain Analysis 14 (2): 49-57.

[2] Hearn, G., and Testa, R. B. 1991. "Modal Analysis for Damage Detection in Structures." Journal of Structural Engineering, ASCE (American Society of Civil Engineers) 117 (10): 3042-63.

[3] Hassiotis, S., and Jeong, G. D. 1993. "Assessment of Structural Damage from Natural Frequency Measurements." Computers and Structures 49 (4): 679-91.
[4] Bicanic, N., and Chen, H. P. 1997. "Damage Identification in Framed Structures Using Natural Frequencies." International Journal for Numerical Methods in Engineering 40 (23): 4451-68.

[5] Hassiotis, S. 2000. "Identification of Damage Using Natural Frequencies and Markov Parameters." Computers and Structures 74 (3): 365-73.

[6] Shi, Z. Y., Law, S. S., and Zhang, L. M. 2000. "Damage Localization by Directly Using Incomplete Mode Shapes." Journal of Engineering Mechanics 126 (6): 656-60.

[7] Reynders, E., Teughels, A., and de Roeck, G. 2010. "Finite Element Model Updating and Structural Damage Identification Using OMAX Data." Mechanical Systems and Signal Processing 24 (5): 1306-23.

[8] Ko, J. M., Sun, Z. G., and Ni, Y. Q. 2002. "Multi-stage Identification Scheme for Detecting Damage in Cable-Stayed Kap Shui Mun Bridge." Engineering Structures 24 (7): 857-68.

[9] Mekjavić, I. 2004. "Durability Assessment of Concrete Bridges Based on Dynamic Parameters." PhD dissertation, University of Zagreb.

[10] Mekjavić, I. 2013. "Damage Identification of Bridges from Vibration Frequencies.” Technical Gazette 20 (1): 155-60.

[11] Herlufsen, H., Andersen, P., Gade, S., and Moller, N. 2005. "Identification Techniques for OMA-An Overview and Practical Experiences." In Proceedings of the 1st IOMAC (International Operational Modal Analysis Conference), Copenhagen, 242-54.

[12] Brincker, R., Zhang, L., and Andersen, P. 2001. "Modal Identification of Output-Only Systems Using Frequency Domain Decomposition." Smart Materials and Structures 10: 441-5.

[13] Jacobsen, N. J., Andersen, P., and Brincker, R. 2006. "Using Enhanced Frequency Domain Decomposition as a Robust Technique to Harmonic Excitation in Operational Modal Analysis." Presented at the 23rd ISMA (International Conference on Noise \& Vibration Engineering), Leuven, Belgium.

[14] Jacobsen, N. J., Andersen, P., and Brincker, R. 2008. "Applications of Frequency Domain Curve-Fitting in the EFDD Technique." Presented at the 26th IMAC: Conference and Exposition on Structural Dynamics, Orlando, USA.

[15] Damjanović, D., and Herceg, L. 2013. Report on Inspection and Testing of Saint Ivan Bridge near Slunj. Faculty of Civil Engineering, University of Zagreb, Croatia. 